\title{
BMJ Open Optimal duration of dual antiplatelet therapy following percutaneous coronary intervention: protocol for an umbrella review
}

\author{
Jesse Elliott, ${ }^{1}$ Shannon E Kelly, ${ }^{1}$ Zemin Bai, ${ }^{1}$ Wenfei Liu, ${ }^{1}$ Becky Skidmore, ${ }^{2}$ \\ Michel Boucher, ${ }^{3}$ Derek Y F So, ${ }^{4}$ George A Wells ${ }^{1}$
}

To cite: Elliott J, Kelly SE, Bai Z, et al. Optimal duration of dual antiplatelet therapy following percutaneous coronary intervention: protocol

for an umbrella review. BMJ Open 2017;7:e015421. doi:10.1136/bmjopen-2016015421

- Prepublication history and additional material is available. To view please visit the journal (http://dx.doi.org/ 10.1136/bmjopen-2016015421)

Received 2 December 2016 Revised 30 January 2017 Accepted 14 February 2017

CrossMark

For numbered affiliations see end of article.

Correspondence to Professor George A Wells; gawells@ottawaheart.ca

\section{ABSTRACT \\ Introduction: Although dual antiplatelet therapy} (DAPT) is routinely given to patients after percutaneous coronary intervention $(\mathrm{PCl})$ with stenting, the optimal duration is unknown. Recent evidence indicates there may be benefits in extending the duration beyond 12 months but such decisions may increase the risk of bleeding. Our objective is to provide a comprehensive overview of the literature for clinicians and policymakers via an umbrella review assessing the optimal duration of DAPT.

Methods and analysis: We will perform a comprehensive search of the published and grey literature for systematic reviews involving randomised controlled trials (RCTs) assessing the optimal duration of DAPT following PCI with stenting. The intervention of interest is extended DAPT (beyond 12 months) compared with short-term DAPT (6-12 months). Studies will be selected for inclusion by two reviewers, and the quality will be assessed. The primary outcomes of interest are all-cause mortality and cardiovascular mortality. Secondary outcomes will be bleeding (major, minor and gastrointestinal), urgent target vessel revascularisation, major adverse cardiovascular events, myocardial infarction, stroke and stent thrombosis. Outcomes will be assessed while on DAPT and after withdrawal of DAPT. Data will be summarised with respect to the number of included RCTs, number of participants, effect estimates and heterogeneity. Data will be reported separately based on patient demographics, procedural parameters (eg, stent types, lesion complexity and concurrent disease) and clinical presentation (eg, acute coronary syndromes, infarct type).

Ethics and dissemination: Our umbrella review aims to provide a comprehensive overview of the benefits and harms associated with extending DAPT beyond 12 months following PCl with stenting. The results of this review will inform clinical and policy decisions regarding the optimal treatment duration and reimbursement of DAPT following $\mathrm{PCl}$ with stenting. Results will be disseminated through a peer-reviewed publication and conference presentations. Ethics approval is not required for this study.

Trial registration number: CRD42016047735.
Strengths and limitations of this study

- This protocol was designed following guidelines for umbrella reviews from Joanna Briggs Institute and reported following the Preferred Reporting Items for Systematic review and Meta-Analyses (PRISMA) guidelines.

- The results of this review will inform policy decisions regarding the reimbursement of DAPT following $\mathrm{PCl}$ with stenting and will be useful for shared decision-making, providing information to clinicians and patients about the potential tradeoffs between risks and benefits of extended DAPT.

- As with other umbrella reviews, the quality of our findings will depend on the quality of the included systematic reviews.

\section{BACKGROUND}

Dual antiplatelet therapy (DAPT), the combination of a P2Y12 inhibitor with acetylsalicylic acid (ASA), is routinely given following percutaneous coronary intervention (PCI) with stenting with the aim of preventing stent thrombosis and major adverse cardiovascular events. ${ }^{1}$ Guidelines currently recommend for DAPT for 6-12 months; ${ }^{1-3}$ however, the optimal duration of DAPT remains controversial. DAPT for $<6$ months may be appropriate in some clinical settings, such as in patients with low risk of stroke but high risk of bleeding, while DAPT for more than 12 months may be appropriate for patients with high risk of stroke and low risk of bleeding. ${ }^{1}$ Extending DAPT beyond 12 months may reduce the risk of late stent thrombosis but may increase the risk of bleeding. ${ }^{4}$ Clinicians must balance the potential risks and benefits in determining the optimal duration of DAPT, and, in some jurisdictions, reimbursement of P2Y12 inhibitors after PCI may be limited to 12 months by some public payers. 
The association between DAPT duration and clinical outcomes has been assessed in many systematic reviews and meta-analyses, ${ }^{5-8}$ yet the optimal duration of DAPT remains a controversial topic. Previous meta-analyses have reported an increased risk of death among patients who received DAPT for more than 12 months following stenting, yet extending DAPT beyond 12 months may reduce the risk of myocardial infarction (MI) and stent thrombosis. ${ }^{6} \quad 7$ These potential benefits must be balanced against an increased risk of harms, including death and major bleeding. Clinicians and policymakers require a comprehensive overview of the depth and strength of the evidence base in order to evaluate the potential benefits and harms associated with extending DAPT beyond 12 months after stenting. To this end, we will perform a comprehensive umbrella review to collect and assess information from previous systematic reviews that have investigated the optimal duration of DAPT following PCI with stenting. We will seek to answer the following questions using the findings of high-quality systematic reviews: What are the benefits and harms of extended DAPT ( $>12$ months vs $6-12$ months) following PCI with stenting? Are there subgroups of patients based on demographics, angiographic parameters or clinical presentation for whom the optimal duration of DAPT is different? Does the optimal duration of DAPT depend on the type of stent implanted (bare-metal stent (BMS) or drug-eluting stent (DES))? Is there a rebound effect after withdrawal of DAPT?

Umbrella reviews are syntheses of existing systematic reviews and/or meta-analyses and provide an ideal method to comprehensively review the evidence base and to explore the contradictory findings of previous reviews. ${ }^{9}$ Since a number of previous systematic reviews on this topic are available and timely evidence is required to inform health policy, undertaking a de novo systematic review would not be appropriate. An umbrella review design will allow us to explore the reasons for discrepant findings in previous systematic reviews and to provide clinicians and policymakers with evidence in a timely manner.

\section{METHODS AND ANALYSIS}

This umbrella review was designed using the methodology guidelines for umbrella reviews provided by the Joanna Briggs Institute. ${ }^{9}$ As well, we followed the Preferred Reporting Items for Systematic review and Meta-Analyses (PRISMA) guidelines ${ }^{10}$ and the extension for protocols. ${ }^{11}$ The completed PRISMA-P checklist is available (see online supplementary file 1 ). This protocol is registered with PROSPERO (no. CRD42016047735).

\section{Search strategy}

We will search for systematic reviews that included randomised controlled trials (RCTs) evaluating different durations of DAPT following PCI with stenting. The search strategy (see online supplementary file 2) was developed and tested by an experienced medical information specialist using an iterative process in consultation with the review team. The search strategy has been reviewed by another senior information specialist using the Peer Review of Electronic Search Strategies (PRESS) checklist. ${ }^{12}$ Embase and Ovid MEDLINE, including Epub Ahead of Print and In-Process and Other Non-Indexed Citations, will be searched using the OVID platform. We will also search the Cochrane Library on Wiley and PubMed for the most recent and unindexed citations. Grey literature from major health technology assessment sources and clinical practice guidelines sources will be searched using CADTH's Grey Matters Light. ${ }^{13}$

The search strategies involve a combination of controlled vocabulary (eg, 'Stents', 'Percutaneous Coronary Intervention', 'Prasugrel Hydrochloride') and keywords (eg, 'DES', 'PCI', 'Clopidogrel'). Vocabulary and syntax will be adjusted across databases. Owing to the depth of the literature base and the timeline of the project, results will be limited to those published between 2011 and August 2016.

\section{Inclusion criteria}

We set the inclusion criteria for this umbrella review following the PICO (population, intervention, comparison, outcome) criteria:

Population: Adult patients who have undergone PCI with any type of stent and who are receiving DAPT. Patients receiving DAPT in the absence of stenting are beyond the scope of this review.

Intervention: DAPT following PCI with stenting for an extended duration (more than 12 months). DAPT may involve any type of P2Y12 inhibitor (clopidogrel, prasugrel and ticagrelor) in combination with ASA.

Comparison: DAPT for 6-12 months. The comparison of $<6$ months of DAPT to $>12$ months of DAPT is beyond the scope of this review.

Outcomes: The primary outcomes of interest are allcause mortality and cardiovascular mortality. Secondary outcomes will be urgent target vessel revascularisation, major adverse cardiovascular events, MI, stroke, stent thrombosis and major, minor and gastrointestinal bleeding, as defined by the individual study protocols and/or publications. A range of bleeding classifications and definitions are expected (eg, TIMI, BARC, GUSTO, REPLACE).

All outcomes will be assessed based on the definitions applied in the systematic reviews, with the exception of major bleeding and major adverse cardiovascular events (depending on components of the composite). Studies will not be included or excluded on the basis of reported outcomes.

\section{Study designs}

Systematic evidence syntheses that included RCTs compared different durations of DAPT following PCI are eligible for inclusion. To be eligible for inclusion, studies must have used a systematic process to the literature 
search and study selection. If RCTs and non-randomised studies are included, the effect estimates from RCTs must be reported separately. If a potentially relevant systematic review includes RCTs that enrolled patients with stent and patients without stent, data must be reported separately for patients with stents and patients without stent. Individual RCTs may be eligible for inclusion to address questions about subgroups if at least $85 \%$ of patients received either a DES or BMS. Data will not be extracted from systematic reviews that included RCTs that enrolled $<85 \%$ patients with stents.

No language restrictions will be used during the screening or study selection process. Owing to the short timeline of this project, we will extract data only from foreign-language studies that can be translated within the timeline of the project. All foreign language studies eligible for inclusion will be listed in an appendix to the final publication.

Since our aim is to summarise data from high-quality systematic reviews, studies must meet the following criteria in addition to the PICO criteria. First, studies must report using a comprehensive search strategy involving two or more electronic databases; second, they must provide an explicit statement describing the inclusion criteria applied to candidate RCTs and third, they must have critically appraised the quality and/or risk of bias of the included RCTs and report the outcome of that process.

\section{Study selection}

The eligibility criteria will be applied to each title and abstract identified in the literature search by two independent reviewers in a standardised manner. All records identified by at least one author as potentially relevant will be obtained in full-text format. The eligibility criteria will then be applied to the full-text records, and a final decision made for inclusion. Conflicts will be resolved by discussion. The reviewers will not be blinded to study authors or centre of publication prior to study selection. Study screening and assessment of eligibility will be facilitated and standardised through the use of DistillerSR (Evidence Partners), an online systematic review software.

\section{Quality assessment}

Two independent reviewers will apply the AMSTAR (A MeaSurement Tool to Assess systematic Reviews) ${ }^{14}$ checklist to each included study, and any disagreements will be resolved by consensus. Only reviews meeting a minimum quality threshold will be included in the summary of findings. We will not use a numerical score on the AMSTAR checklist to define 'high quality'; instead, the following criteria must be met: use of a comprehensive search strategy involving two or more electronic databases; use of an explicit statement describing the inclusion criteria applied to candidate RCTs; use of a formal critical appraisal or quality assessment process for all included studies and report the outcome of that process; report findings on outcomes of interest using details on the study and patient characteristics of two or more studies and provide the direction of the findings from any pooled analyses (narrative or meta-analysis) carried out, including direction of effect and any statistical significance. Any included reviews that do not meet these minimum requirements will remain included; however, no data will be extracted.

In the event that included reviews report significantly overlapping lists of included studies reporting the same outcome(s), we will report findings from the higher quality, more recent review with the largest number of studies.

\section{Data collection}

All information will be collected using piloted and standardised data abstraction forms in DistillerSR. Extraction forms will be developed following the Joanna Briggs Institute's recommended extraction items. ${ }^{9}$ Data will be extracted from each included systematic review by one reviewer and verified by a second reviewer. Any disagreements will be resolved by consensus when possible; otherwise, the judgement of a third reviewer will be considered final. The original, primary publication for each included review will be used for data extraction, except where multiple publications for a unique review are found. Multiple publications for a unique review (eg, see supplementary online appendices, companion publications of specific outcomes or populations from the original study) will be handled by extracting the most recently adjudicated data for each outcome specified a priori in this protocol.

The data extracted will include specific details about the included RCTs (eg, study population, the durations of DAPT investigated) and the review methods (eg, number of databases searched, search date and any date, location or language restrictions on the search). Patient characteristics (eg, age, sex, smoking status, diabetes, prior MI, history of heart failure) will also be extracted from the included reviews, if reported. We plan to extract the effect estimates for the outcomes of interest for the whole population and for any subgroups, as well as the method of synthesis (eg, meta-analysis, network meta-analysis). The authors' overall conclusion or recommendation will also be extracted. Outcome data will be extracted for the period while patients were on DAPT and after withdrawal of DAPT.

\section{Subgroups}

If available, effect estimates will be extracted separately for clinically important subgroups based on patient demographics (eg, age, sex, smoking status, diabetes, prior MI, history of heart failure), procedural parameters (eg, vein graft intervention, left main intervention, stent type, lesion complexity, concurrent disease) and clinical presentation (eg, acute coronary syndrome (ACS) vs no ACS; ST-elevation MI (STEMI) vs non-STEMI). If subgroup data are not available from the 
included SRs, we will extract such data from the RCTs identified from the included SRs.

\section{Data summary}

The aim of this umbrella review is to present a summary of the existing research syntheses that have addressed the optimal duration of DAPT. The findings will be summarised from the most recent high-quality systematic reviews using a narrative approach. A tabular summary of review characteristics (year of publication, county of origin, number of included studies, setting and/or context and interventions) will be provided. Outcome data will be summarised with respect to the number of included studies, number of participants, effect estimates and heterogeneity. Data for all bleeding outcomes will be summarised as appropriate using the similarity of outcome definitions and classification systems to guide synthesis and/or pooling. Data for subgroups will be presented separately. Strengths and limitations of the included studies, as assessed by AMSTAR, will also be presented.

\section{DISSEMINATION}

In this umbrella review, we will undertake a comprehensive review of previously published systematic reviews assessing the optimal duration of DAPT. Using evidence from this review, we expect to make a conclusion regarding the benefits and harms associated with extending DAPT beyond 12 months following PCI with stenting. As well, we aim to determine whether there are subgroups of patients who would benefit from shorter or longer DAPT. The results of our review will be of interest to clinicians, policymakers and patients. We plan to disseminate our findings through peer-reviewed journal publication and conference presentations. This review does not require ethical approval.

\section{Author affiliations}

${ }^{1}$ Cardiovascular Research Methods Centre, University of Ottawa Heart Institute, Ottawa, Ontario, Canada

${ }^{2}$ Independent Contractor, Ottawa, Ontario, Canada

${ }^{3}$ Canadian Agency for Drugs and Technologies in Health (CADTH), Ottawa, Ontario, Canada

${ }^{4}$ Division of Cardiology, University of Ottawa Heart Institute, Ottawa, Ontario, Canada

Contributors JE, SEK, MB, DYFS and GAW designed the study. BS designed and will execute the search strategy. JE drafted the protocol, which was revised by all authors. All authors have approved the version of the manuscript submitted for publication.
Funding This work is funded by the Canadian Institutes of Health Research, Drug Safety and Effectiveness Network. The funder had no role in the design of the study.

Competing interests None declared.

Provenance and peer review Not commissioned; externally peer reviewed.

Open Access This is an Open Access article distributed in accordance with the Creative Commons Attribution Non Commercial (CC BY-NC 4.0) license, which permits others to distribute, remix, adapt, build upon this work noncommercially, and license their derivative works on different terms, provided the original work is properly cited and the use is non-commercial. See: http:// creativecommons.org/licenses/by-nc/4.0/

\section{REFERENCES}

1. Levine GN, Bates ER, Mauri L, et al. 2016 ACC/AHA guideline focused update on duration of dual antiplatelet therapy in patients with coronary artery disease. A report of the American College of Cardiology/American Heart Association Task Force on clinical practice guidelines. Circulation 2016;133:e123-55.

2. Windecker S, Kolh P, Alfonso F, et al., Authors/Task Force members. 2014 ESC/EACTS Guidelines on myocardial revascularization. Eur Heart $J$ 2014;35:2541-619.

3. Tanguay JF, Bell AD, Ackman ML, et al. Focused 2012 update of the Canadian cardiovascular society guidelines for the use of antiplatelet therapy. Can J Cardiol 2013;29:1334-45.

4. Mauri L, Kereiakes DJ, Yeh RW, et al. Twelve or 30 months of dual antiplatelet therapy after drug-eluting stents. $N$ Engl J Med 2014;371:2155-66.

5. Liu M, Chen J, Huang D, et al. Optimal duration of dual antiplatelet therapy after drug-eluting stent implantation: a meta-analysis of 3 randomized controlled trials. J Cardiovasc Pharmacol 2014;64:41-6.

6. Navarese EP, Andreotti F, Schulze V, et al. Optimal duration of dual antiplatelet therapy after percutaneous coronary intervention with drug eluting stents: meta-analysis of randomised controlled trials. BMJ 2015;350:h1618.

7. Fei Y, Tsoi MF, Cheung TT, et al. Optimal duration of dual antiplatelet therapy after drug-eluting stent implantation: meta-analysis of randomized controlled trials. Int J Cardiol 2016;220:895-900.

8. Palmerini T, Sangiorgi D, Valgimigli M, et al. Short- versus long-term dual antiplatelet therapy after drug-eluting stent implantation: an individual patient data pairwise and network meta-analysis. J Am Coll Cardiol 2015;65:1092-102.

9. Aromataris E, Fernandez R, Godfrey C, et al. Methodology for JBI Mixed Methods Systematic Reviews. 2014. http://joannabriggs.org/ assets/docs/sumari/ReviewersManual-Methodology-JBI UmbrellaReviews-2014.pdf (accessed15 Aug 2016).

10. Moher D, Liberati A, Tetzlaff J, et al. Preferred reporting items for systematic reviews and meta-analyses: the PRISMA Statement (Reprinted from Annals of Internal Medicine). Phys Ther 2009;89:873-80.

11. Moher D, Shamseer L, Clarke M, et al. Preferred Reporting Items for Systematic Review and Meta-Analysis Protocols (PRISMA-P) 2015 statement. Syst Rev 2015;4:1.

12. McGowan J, Sampson M, Lefebvre C. An evidence based checklist for the Peer Review of Electronic Search Strategies (PRESS EBC). Evid Based Libr Inf Pract 2010;5:149-54.

13. Canadian Agency for Drugs and Technologies in Health. Grey matters light. http://www.cadth.ca/sites/default/files/is/cadth Handout_greymatters_light_e.pdf (accessed 15 Aug 2016).

14. Shea BJ, Grimshaw JM, Wells GA, et al. Development of AMSTAR: a measurement tool to assess the methodological quality of systematic reviews. BMC Med Res Methodol 2007;7:10. 